\title{
Lattice Study of the Extent of the Conformal Window in Two-Color Yang-Mills Theory
}

\section{Gennady Voronov* (for the LSD Collaboration)}

Department of Physics, Yale University, New Haven, CT 06511, USA

E-mail: gennady.voronov@yale.edu

We perform a lattice calculation of the Schrödinger functional running coupling in $S U(2)$ YangMills theory with six massless Wilson fermions in the fundamental representation. The aim of this work is to determine whether the above theory has an infrared fixed point. Due to sensitivity of the $S F$ renormalized coupling to the tuning of the fermion bare mass we were unable to reliably extract the running coupling for stronger bare couplings.

XXIX International Symposium on Lattice Field Theory

July 10 - 162011

Squaw Valley, Lake Tahoe, California

${ }^{*}$ Speaker. 


\section{Introduction}

A SM Higgs adequately accounts for all electroweak measurements (for now). However, it has a number of theoretical shortcomings, chiefly among them the hierarchy problem. Technicolor is a promising alternative for electroweak symmetry breaking that avoids the introduction of a fundamental light scalar particle. In particular, a Technicolor model based on a walking gauge theory can properly account for the Standard Model fermion masses[1]. Since such a theory is expected to reside just below the conformal window, it is essential to narrow down the extent of this window. The goal of this work is to do that for the $S U(2)$ gauge theories with $N_{f}$ fermion flavors in the fundamental representation. These gauge theories are special in that there is an enhanced $S U\left(2 N_{f}\right)$ global symmetry and it is currently unknown what implications this will have for technicolor model building.

The asymptotic properties of a field theory are encoded in the RG flow of the couplings. We are particularly interested in the infrared dynamics of two-color asymptotically free theories. Such theories have two possible distinct IR behaviors. One possibility is that the $\beta$ function has no zero aside from the one that leads to the Gaussian UV fixed point. In this case, the running coupling will increase until it is sufficiently strong to break chiral symmetry. All fermions will screen out and the coupling will run as in the pure gauge theory. Alternatively the $\beta$ function may have an additional zero leading to an IR fixed point.

There exists a large parameter space of Yang-Mills theories. One can consider different gauge symmetries and different numbers of fermion flavors transforming under various representations of the gauge group. Restricting ourselves to $N_{f}$ flavors of fermions transforming under the fundamental representation of $S U\left(N_{c}\right)$ and fixing $N_{c}$, perturbation theory precisely tells us how many fermions flavors are required to maintain asymptotic freedom. For two-colors, asymptotic freedom sets in for $N_{f}<11$. At a fixed color, as we lower the number of fermion flavors to just below where asymptotic freedom sets in, we have a weakly-coupled IR fixed point and therefore an IR conformal theory [2]. However, for small $N_{f}$ we know that chiral symmetry is broken in the IR and the theory is confining. Therefore, we expect that there is some critical number of fermion flavors, $N_{f}^{c}$ at which a theory at fixed color transitions from confining to conformal IR behavior and we can talk of a conformal window, i.e. the range of $N_{f}$ at which the theory is both conformal in IR and asymptotically free in UV.

The $\beta$ function can be expanded in perturbation theory. The first two perturbative coefficients are universal and are given in [2]. The two loop expansion suggests that $5<N_{f}^{c}<6$ and the six flavor theory has an IRFP around $\bar{g}_{*}^{2} \approx 144$. In perturbation theory, the IRFP at the upper end of the conformal window is quite weak. As we proceed in decreasing $N_{f}$, the strength of the perturbative IRFP grows. Therefore, at the lower end of the conformal window perturbation theory is likely unreliable and we will need to narrow down the extent of the conformal window using non-perturbative methods.

The $S U(2)$ gauge theories with $N_{f}$ flavors of fermions in the fundamental representation have been previously studied. The $N_{f}=6$ theory is studied by Bursa et. al. They show evidence that the $N_{f}=6$ theory is consistent with an IRFP $5<\bar{g}_{*}^{2}<6$ (in the SF scheme) [3]. Ohki et. al. show evidence that the $N_{f}=8$ theory has a fixed point $\bar{g}_{*}^{2}>7.5$ using a twisted Wilson/Polyakov loop method in [4]. It is peculiar that the six flavor theory as studied by Bursa et. al. would have a weaker 
fixed point than the eight flavor theory, especially given how strong the six flavor perturbative fixed point is. Our goal is to thoroughly study the $S U(2)$ gauge theories with fermions in the fundamental representation. We would like to narrow down the extent of the conformal window for this class of theories. The unexpected results for the six flavor theory prompted us to begin with it, to see if we could either confirm or refute the result. Since this work was undertaken, Karavirta et. al. presented an $S U$ (2) $S F$ study of the $N_{f}=4,6$, and 10 theories. They find no evidence of an IRFP, inconclusive evidence of an IRFP with $\bar{g}_{*}^{2}>11$, and evidence of an IRFP $\bar{g}_{*}^{2} \approx 1$ in the four, six, and ten flavor theories respectively [5].

\section{Schrödinger Functional Scheme and Step Scaling}

The Schrödinger functional (SF) $\mathscr{Z}$ allows us to define a non-perturbative renormalized coupling [6]; it is given by a path integral over gauge and fermion fields that reside within a fourdimensional Euclidean box of spatial extent $L$ with periodic boundary conditions in spatial directions and Dirichlet boundary conditions in the time direction. We choose gauge boundary conditions [7], $\left.U(x, \mathrm{k})\right|_{x^{0}=0}=\exp \left[-i \eta_{L} \frac{a}{L} \tau_{3}\right]$ and $\left.U(x, \mathrm{k})\right|_{x^{0}=L}=\exp \left[-i(\pi-\eta) \frac{a}{L} \tau_{3}\right]$, and fermion boundary conditions [8], $\left.P_{+} \psi\right|_{x^{0}=0}=\left.\bar{\psi} P_{-}\right|_{x^{0}=0}=\left.P_{-} \psi\right|_{x^{0}=L}=\left.\bar{\psi} P_{+}\right|_{x^{0}=L}=0$. The gauge boundary conditions classically induce a constant chromoelectric background field whose strength is characterized by the dimensionless parameter $\eta$. With these boundary conditions we see that the $\mathrm{SF} \mathscr{Z}(\eta, L)=\int D[U, \psi, \bar{\psi}] e^{-S[U, \psi, \bar{\psi} ; \eta]}$.

The running coupling, in the SF scheme, is defined by,

$$
\frac{k}{\bar{g}^{2}(L)}=\left.\frac{\partial}{\partial \eta} \log \mathscr{Z}\right|_{\eta=\pi / 4}=\left\langle\frac{\partial S}{\partial \eta}\right\rangle,
$$

where $k=-24(L / a)^{2} \sin \left[(a / L)^{2}(\pi-2 \eta)\right]$ is chosen so that the renormalized coupling agrees with the bare coupling at tree-level. Note that the first two perturbative coefficients of the SF running coupling are exactly the universal coefficients given in [2]. We now have a non-perturbative definition for a renormalized coupling in a form that is amenable to a lattice calculation.

For this work we used the standard Wilson plaquette gauge action and the Wilson fermion action. An advantage of working in the SF scheme is that we can evaluate the running coupling along the $m_{c}\left(g_{0}^{2}\right)$ curve. $m_{c}\left(g_{0}^{2}\right)$ is defined as the bare mass value that results in a zero PCAC quark mass [9]. We determined $m_{c}$ for a range of bare coupling on $8^{3} \times 16$ and $16^{3} \times 32$ lattices. We found $m_{c}$ to be consistent within statistics between the two volumes and consequently interpolated an $m_{c}$ curve in the $m_{0}-g_{0}^{2}$ plane using the smaller volume data. For $g_{0}^{2} \leq 0.5$, we determine $m_{c}$ using two-loop perturbation theory [10]. Otherwise, we use the procedure outlined above.

We are interested in investigating the running of the coupling over a large range of scales. A step scaling analysis enables us to do this in a manner that is computationally feasible [11]. We begin by calculating the SF renormalized coupling over a range of bare couplings and lattice volumes. Lattice perturbation theory gives $g_{0}^{2} / \bar{g}^{2}$ as an expansion in powers of $g_{0}^{2}$. This motivates an interpolating fit [12],

$$
\frac{1}{\bar{g}^{2}\left(g_{0}^{2}, \frac{L}{a}\right)}-\frac{1}{g_{0}^{2}}=\sum_{i=0}^{n} \sum_{j=0}^{m} a_{i, j} g_{0}^{2 i}\left(\frac{a}{L}\right)^{j} .
$$




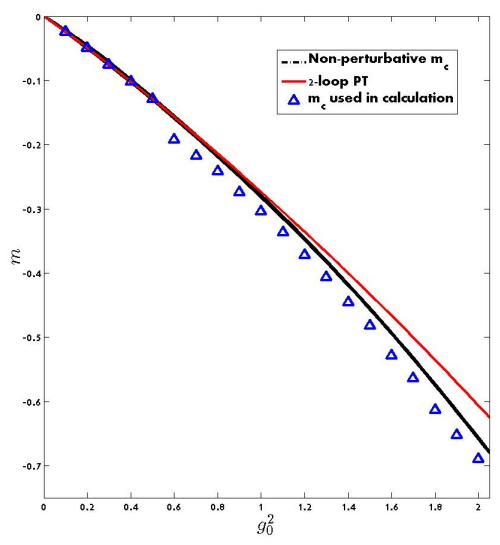

(a)

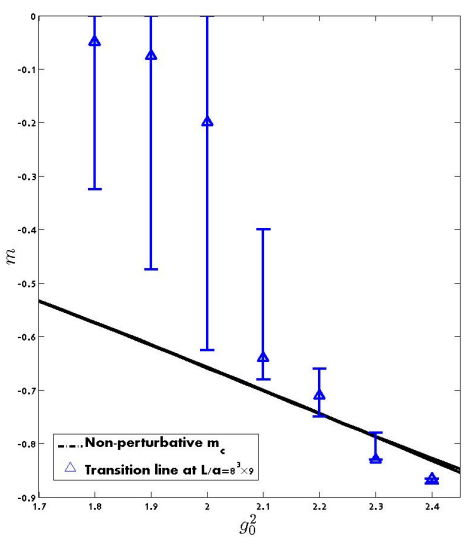

(b)

Figure 1: Critical bare mass to reproduce massless fermions. In (a) continuum extrapolation of $m_{c}$, 2-loop perturbative $m_{c}$, and values used in calculation shown. In (b) continuum $m_{c}$ shown against location of bulk phase transition.

This procedure produces a smooth function of the renormalized coupling versus the bare coupling and inverse lattice volume. We note that it is advantageous to perform one global fit to all data rather than fitting an interpolating polynomial to each lattice volume. This allows us to interpolate to additional lattice volumes and smooths out the approach to the continuum limit. In Figure 2a below we show a bootstrap replication of our renormalized coupling data plotted against a global fit.

Now we define the discrete step scaling function,

$$
\left.\Sigma\left(u, \frac{a}{L}\right) \equiv \bar{g}^{2}\left(g_{0_{*}}^{2}, \frac{s L}{a}\right)\right|_{\bar{g}^{2}\left(g_{0_{*}}^{2}, \frac{L}{a}\right)=u},
$$

it is the value of the renormalized coupling on a volume of $(s L)^{4}$ and bare coupling tuned such that we have a renormalized coupling of $u$ on a lattice of volume $L^{4}$. We arrive at a continuum step scaling function,

$$
\sigma(u)=\lim _{\frac{a}{L} \rightarrow 0} \Sigma\left(u, \frac{a}{L}\right),
$$

by taking the continuum limit of the discrete step scaling function. In practice, these functions are evaluated using the interpolating polynomial specified in Eq. (2.2) and with the choice $s=2$. We take the continuum limit by evaluating $\Sigma$ for various values of $a / L$, taking care to only to use interpolated values of $a / L$, and extrapolating to zero.

\section{Results}

We calculated the renormalized coupling for a range of values of the bare coupling $g_{0}^{2}$ and lattice volumes $L / a$. Configurations were generated with the Chroma implementation of the HMC 


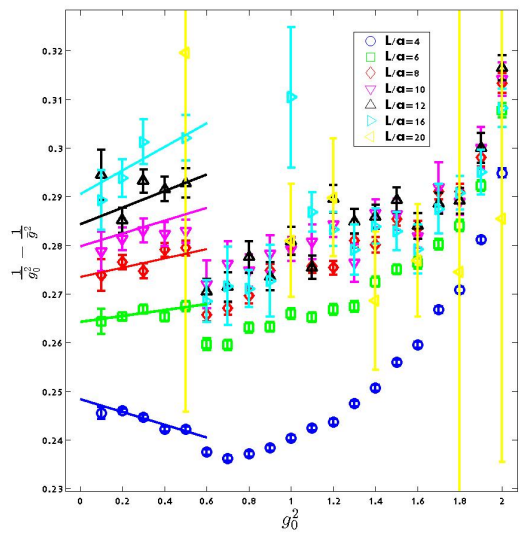

(a)

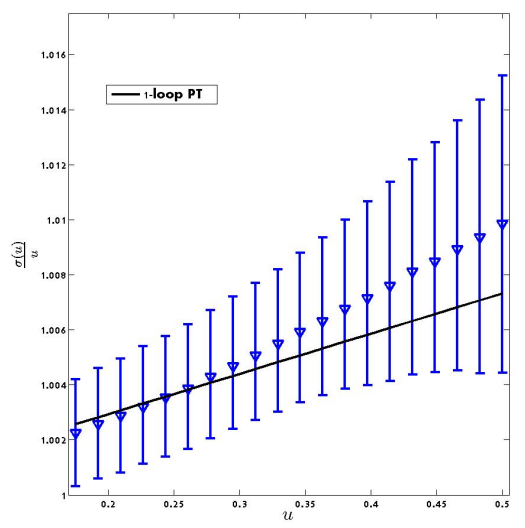

(b)

Figure 2: In (a), all renormalized coupling data points in a typical bootstrap ensemble plotted in form $\frac{1}{g_{0}^{2}}-\frac{1}{\bar{g}^{2}}$ against a global interpolating fit to data restricted to $g_{0}^{2} \leq 0.5$. In (b), a plot of the discrete beta function derived from fit shown in (a).

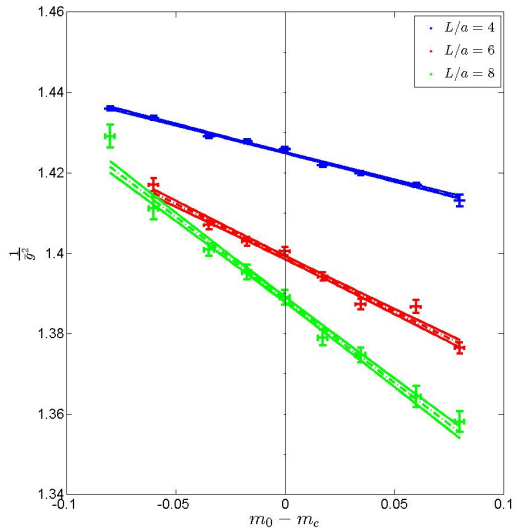

(a)

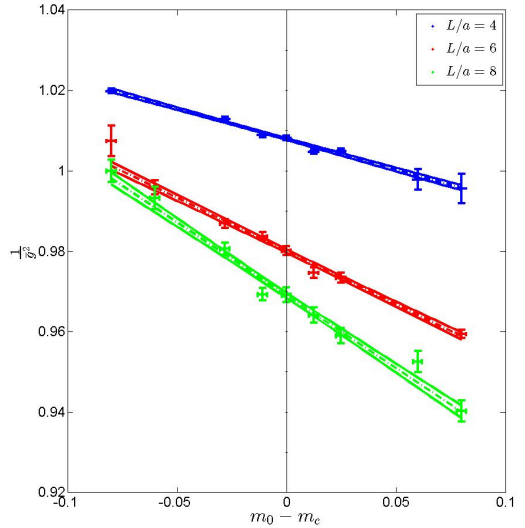

(b)

Figure 3: $\bar{g}^{2}$ evaluated at $g_{0}^{2}=0.6$ and 0.8 , in (a) and (b) respectively, for various lattice volumes and fermion bare masses.

algorithm and $\frac{\partial S}{\partial \eta}$ is evaluated on every configuration [13]. We aimed to acquire on the order of $100 \mathrm{~K}$ configurations since $\frac{\partial S}{\partial \eta}$ is a noisy observable with long autocorrelation times.

Upon studying the mass tuning on additional lattice volumes and obtaining additional statistics, we see that our initial observation of no statistically significant dependence on the lattice volume was incorrect. We calculated an improved $m_{c}$ curve by fitting our non-perturbatively determined critical bare mass values to an interpolating polynomial $m_{c}\left(g_{0}^{2}, \frac{a}{L}\right)$ and extrapolating to $a / L \rightarrow 0$. In Figure 1a, we show the the values of $m_{c}$ used in our simulations, the two-loop perturbative $m_{c}$ curve, and our improved non-perturbative $m_{c}$ curve. It is clear that the values of $m_{c}$ 
used in our evaluations of the renormalized coupling deviate significantly from the appropriate values. Moreover, Figure 2a, which shows our renormalized coupling measurements versus the bare coupling, indicates a jump discontinuity at precisely the value of the bare coupling where we switch from using the perturbatively determined $m_{c}$ to our miss-tuned values of $m_{c}$. We performed a global fit to all of our renormalized coupling data that was calculated with a perturbative value of $m_{c}$, i.e. for $g_{0}^{2} \leq 0.5$. The discontinuity in $\bar{g}^{2}$, is highlighted by extrapolating our fit to the first data points which are mistuned in the bare mass. To quantify this we evaluated a limited sample of renormalized couplings at a variety of bare masses near $m_{c}$. Figure 3 shows $1 / \bar{g}^{2}$ versus $m_{0}$ for various lattice volumes and $g_{0}^{2}=0.6$ and 0.8 . This figure demonstrates that $\bar{g}^{2}$ is trending in the correct direction to remove the discontinuity in Figure $2 \mathrm{a}$ and that a mistuning of the bare mass by as little as $4 \%$ results in an error in the renormalized coupling that dominates all other sources of error. Moreover, such an error can easily introduce or obscure an IRFP, especially if the the RG flow of the running coupling is particularly slow.

In order to guarantee that we can take a continuum limit, we need to ensure that we obtain data from the weak-coupling side of any spurious lattice phase transition. With this in mind, we scanned through the bare parameter space and located peaks in the plaquette susceptibility on a $L / a=8^{3} \times 9$ lattice. This search indicates a line in the $m_{0}-g_{0}^{2}$ plane of first order phase transition that ends at a critical point at around $g_{0}^{2} \approx 2.2$. For $g_{0}^{2} \lesssim 2$.2, we see crossover behavior. In Figure $1 \mathrm{~b}$, we show the above transition line plotted along with $m_{c}\left(g_{0}^{2}\right)$. Figure $1 \mathrm{~b}$ indicates that the six flavor massless fundamental Wilson fermion action has a sensible continuum limit only for $g_{0}^{2} \lesssim 2.2$. Therefore, we expect that we will not be able to examine the running coupling at sufficiently strong bare coupling with our current action.

Finally, using only data generated with a properly tuned bare mass $\left(g_{0}^{2} \leq 0.5\right)$, we study the continuum running of this theory. Towards this end, we generated 2000 bootstrap ensembles from our restricted data set and apply the fitting procedure described in the previous section to each individual ensemble. A step scaling analysis, using fits like the one shown in Fig 2a, with $s=2$, using a linear extrapolation to the continuum using linearly spaced values between $L / a=7$ and 10 , was then used to produce curves, for each individual ensemble, of the discrete beta function $\sigma(u) / u$. Figure $2 \mathrm{~b}$ shows $\sigma(u) / u$ plotted against one-loop perturbation theory. Each point, with two-sided errorbars, was obtained via the $\mathrm{BC}_{\mathrm{a}}$ method [14]. This plot demonstrates, that our methods can properly reproduce perturbation theory when the bare mass is tuned appropriately.

\section{Conclusions and Outlook}

We were unable to extract a renormalized coupling flow outside the perturbative region due to mistuning of the bare fermion mass. We have since properly tuned the bare mass. Figure $2 b$ demonstrates that our production and analysis applications can reproduce perturbation theory in a robust manner. We found that these figures do not qualitatively change as we change the fit parameters and details of the continuum extrapolation.

The RG flow of the running coupling in the six-flavor $S U$ (2) theory is particularly slow and consequently the renormalized coupling sensitively depends on the bare fermion mass. We emphasize that in such theories the bare mass must be tuned very carefully. We have since done this for 
the $S U$ (2) six-flavor theory. In order to reliably study the running coupling of this theory we would simply have to reproduce our data using the correct value of the fermion bare mass.

Studies of the plaquette and plaquette susceptibility indicate the presence of bulk phase transition along the $m_{c}$ line at $g_{0}^{2} \approx 2.2$. This effectively limits how strong of a renormalized coupling that we can investigate with the standard Wilson fermion action. Other investigations into the sixflavor theory suggest that if there exists an IRFP then it likely resides at a stronger coupling that can be probed with our current action before encountering a bulk phase transition [5]. Therefore, while we can regenerate a new data set with an improved estimate of $m_{c}$, we believe that it would be a misallocation of resources to pursue this theory without first switching to an improved action where the bulk phase transition occurs at a stronger coupling.

\section{References}

[1] T. Appelquist, D. Karabali, L. Wijewardhana, Chrial hierarchies and the flavor changing neutral current problem in technicolor, Phys. Rev. Lett. 57 (1986) 957.

[2] W. Caswell, Asymptotic behavior of non-abelian gauge theories to two-loop order, Phys. Rev. Lett. 33 (1974) 244.

[3] F. Bursa, L. Del Debbio, L. Keegan, C. Pica and T. Pickup, Mass anomalous dimension and running of the coupling in SU(2) with six fundamental fermions, PoS Lattice2010 (2010) 070 [arXiv:1010.0901].

[4] H. Ohki, Study of the scaling properties in SU(2) gauge theory with eight flavors, PoS Lattice2010 (2010) 066 [arXiv:1011.0373].

[5] T. Karavirta, J. Rantaharju, K. Rummukainen and K. Tuominen, Determining the conformal window: $S U(2)$ gauge theory with $N_{f}=4,6$ and 10 fermion flavors, arXiv:1111.4104.

[6] M. Lüscher, R. Narayanan, P. Weisz and U. Wolff, The Schrödinger functional - a renormalizable probe for non-abelian gauge theories, Nucl. Phys. B384 (1992) 168-228 [arXiv:hep-lat/9207009].

[7] M. Lüscher, R. Sommer, U. Wolff and P. Weisz, Computation of the running coupling in the SU(2) Yang-Mills theory, Nucl. Phys. B389 (1993) 247-264 [arXiv:hep-lat/9207010].

[8] S. Sint, On the Schrödinger functional in QCD, Nucl. Phys. B421 (1994) 135-158 [arXiv:hep-lat/9312079].

[9] M. Lüscher, S. Sint, R. Sommer, P. Weisz and U. Wolff, Non-perturbative $O($ a) improvement of lattice QCD, Nucl. Phys. B491 (1997) 323-343 [arXiv:hep-lat/9609035].

[10] H. Panagopoulos and Y. Proestos, The critical hopping parameter in $\mathscr{O}(a)$ improved Lattice QCD, Phys. Rev. D65 (2002) 014511 [arXiv:hep-lat/0108021].

[11] M. Lüscher, P. Weisz and U. Wolff, A numerical method to compute the running coupling in asymptotically free theories, Nucl. Phys. B359 (1991) 221-243.

[12] T. Appelquist, G. Fleming, and E. Neil, Lattice study of conformal behavior in SU (3) Yang-Mills theories, Phys. Rev. D79 (2009) 076010 [arXiv:0901.3766].

[13] R. Edwards and B. Joó, The Chroma software system for lattice QCD, Nucl. Phys. Proc. Suppl. 140 (2005) 832 [arXiv:hep-lat/0409003].

[14] B. Efron, R. Tibshirani, An introduction to the bootstrap, Chapman \& Hall, Boca Raton 1993. 